\title{
Reliability of Local Activation Waves Features to Characterize Paroxysmal Atrial Fibrillation Substrate During Sinus Rhythm
}

\author{
Aikaterini Vraka ${ }^{1}$, Fernando Hornero ${ }^{2}$, Aurelio Quesada ${ }^{3}$, Luca Faes ${ }^{4}$, Raúl Alcaraz ${ }^{5}$ José J Rieta $^{1 *}$ \\ ${ }^{1}$ BioMIT.org, Electronic Engineering Department, Universitat Politecnica de Valencia, Spain \\ ${ }^{2}$ Cardiovascular Surgery Department, Hospital Clínico Universitario de Valencia, Spain \\ ${ }^{3}$ Cardiology Department, Hospital General Universitario de Valencia, Spain \\ ${ }^{4}$ Department of Engineering, University of Palermo, Palermo, Italy \\ ${ }^{5}$ Research Group in Electronic, Biomed. and Telecomm. Eng., Univ. of Castilla-La Mancha, Spain
}

\begin{abstract}
Analysis of coronary sinus (CS) electrograms (EGMs) is vastly used for the assessment of the atrial fibrillation $(A F)$ substrate. As a catheter consists of five dipoles (distal, mid-distal, medial, mid-proximal, proximal), results may vary upon the employed channel: myocardial contraction and bad contact are unavoidable factors affecting the recording. This work aims to specify the most reliable channels in catching AF dynamics, using 44 multichannel bipolar CS recordings in sinus rhythm (SR) of paroxysmal AF with 1-5 minutes duration. Local activation waves (LAWs) were detected and main features obtained: duration, amplitude, area and correlation between dominant morphologies of each channel. Analysis was performed with Kruskal-Wallis test for multichannel comparison and Mann-Whitney U-test for pairs of channels and comparison between one and the remaining channels, using Bonferroni correction. Median values were calculated. Distal channel presented the highest alteration in LAWs features, being the least correlated channel (82.84-88.31\%) with the lowest amplitude and area $\left(p_{\max }<0.01\right)$. Contrastly, medial and mid-proximal channels showed the most robust LAW characteristics, with very high correlation $(94.53 \%)$ and high area and amplitude values $\left(p_{\max }<0.02\right.$ and $p_{\max }<0.07$, respectively) and their analysis is recommended for AF substrate characterization during SR.
\end{abstract}

\section{Introduction}

Atrial fibrillation (AF) is the cardiac arrhythmia with the highest global burden that is accompanied with significant health risks [1]. Catheter ablation (CA) of pulmonary veins (PVs) is the premier AF treatment [2], especially for paroxysmal AF patients. During CA of PVs, diagnostic catheters are inserted in other cardiac sites for pacing, stimulation and signal recording [3]. Due to the vicinity with both right and left atrium, coronary sinus (CS) has been proven to form interatrial electrical connection $[4,5]$. Consequently, CS recordings are vastly used for the assessment of the AF substrate [3, 6-9]. Previous studies investigating CS recordings during sinus rhythm (SR) or AF report cases of spontaneous initiation of atrial tachycardia leading to $\mathrm{AF}[10,11]$ or attribute $\mathrm{AF}$ perpetuation to $\mathrm{CS}$ musculature $[7,12,13]$.

The most frequent CS catheter used is a decapolar catheter with its most proximal electrode placed to the CS ostium and its most distal electrode placed proximally to the left atrium (LA) area [3]. Variable CS shape and vigorous myocardial contraction require delicate movements for proper catheter insertion and advancement, while catheter dislocation during CA is not an exceptional case [14].

The aforementioned factors may affect the quality of CS electrograms (EGMs) in any of its channels. Atrial remodelling issues may affect additionally the CS anatomy [15], thus complicating even more the robust recording of $\mathrm{AF}$ dynamics. Hence, in order to precisely record and detect any possible changes occurred in CS due to CA process, the channels providing the most and least alterations on AF dynamics need to be specified. The following study aims to define these channels acquired from CS recordings of CA during SR in order to assist future studies focused on AF substrate mappig.

\section{Materials and Methods}

Fourty-four multichannel bipolar recordings of 28 paroxysmal AF patients undergoing radiofrequency (RF) CA for the first time were used. Recordings were in SR, lasted from 60 to 300 seconds and were performed before and after CA with a decapolar catheter with sampling frequency of $1 \mathrm{kHz}$ which was inserted through right femoral vein. Decapolar catheter consists of five dipoles corresponding to five EGM channels: distal, mid-distal, medial, 
mid-proximal and proximal. Distal is the channel recorded from the dipole in the tip of the catheter and is the closest channel to the left atrium (LA).

When present, ventricular contamination was removed by localizing and blanking the activity that corresponded to the QRS interval of the surface electrocardiograms (ECGs). After ventricular cancellation, EGMs were denoised by a wavelet-denoising technique [16] and the mean was removed.

Local activation waves (LAWs) [17] were detected with the help of an alternative Botteron's technique making use of a hyperbolic tangent function [18]. Each LAW was extracted to a segment of $201 \mathrm{~ms}$ with the sample point corresponding to the activation centered and the following characteristics were calculated: duration, maximum, minimum and peak-to-peak (PP) amplitude, root mean square (RMS) value, positive and negative area, number of deflections and inflections (NODI) of the main LAW area. The aforementioned features are described in the following section.

\subsection{LAW duration}

LAW was smoothed by a 5-point moving average filter and transformed into a normalized curve from 0 to 1 according to an alternative Botteron's technique [18]. The onset was set at the first sample point of amplitude greater than $0.1 \mathrm{mV}$ of the normalized curve. Respectively, the offset was detected as the last sample point to have an amplitude higher than $0.1 \mathrm{mV}$. Fiducial points of the original signal were the same as in the normalized curve. Duration was calculated as the distance between onset and offset.

\subsection{LAW amplitude}

For maximum and minimum amplitude, the highest positive and negative peaks were found, respectively. PP amplitude was the distance between maximum and minimum amplitude. RMS value was also calculated.

\subsection{LAW area}

In order to calculate the positive LAW area, the zerocrossing points were firstly detected and the intervals corresponding to positive LAW values were integrated. The same process was followed for the calculation of the negative LAW area, where integration was performed for the negative LAW values. Final positive and negative LAW areas were the sum of the positive and negative segments, respectively.

\subsection{Number of deflections and inflections}

Since the main objective of this task was to define the prominent NODI of high amplitude, the original signal was normalized from -1 to 1 and two auxiliary baselines at \pm 0.25 of the maximum amplitude were created. Points crossing the auxiliary baselines were found and stored in a list classified according to the sign of the slope that they belong to, so that points belonging to deflections were stored as " 1 " and points belonging to inflections were stored as " 2 ". In order to avoid double counting, if two adjacent points in the list with the same code were detected, the second one was deleted.

\subsection{Correlation of dominant morphology}

For each channel, the most representative LAW was defined with the help of a reference signal created by the mean of the 20 most correlated LAWs. Correlation between the dominant morphologies of different channels of the same recording were further calculated with the help of an Adaptive Signed Correlation Index (ASCI) with a tolerance of $12 \%$ [19] and the median ASCI value for each pair of channels was computed.

\subsection{Statistical analysis}

For all features, multichannel comparison was performed using Kruskal-Wallis (KW) test. Any significant differences between two channels were furthermore explored by Mann-Whitney U-test (MWUt). The latter, with Bonferroni correction, was also applied in order to define if any of the channels presented significant differences from the rest at any of the analyzed features. For this purpose, the median values of each channel were also calculated.

\section{Results}

KW analysis showed that duration, all amplitude and area features differ significantly among channels. MWUt in pairs of two channels demonstrated that differences in the aforementioned features were mostly located between the distal $\backslash$ mid-distal channels and the medial $\backslash$ midproximal channels. No statistical differences were found in NODI when all channels or combination of pairs of channels where analyzed. Table 1 shows the $p$-values marking a statistical difference in these channels for all the features. Further statistical differences where found between distal and proximal channels in maximum amplitude $(p=0.0430)$ and between medial and proximal channels in duration $(p=0.0462)$.

Comparison of each one with the remaining channels revealed that distal channel showed the lowest amplitude and area values, while the medial channel showed the highest values in positive amplitude and area and RMS value and a trend for the highest values in the remaining amplitude and area features ( $p=0.012-0.018)$. Due to Bonferroni correction, there was a trend for longer LAW duration in distal 


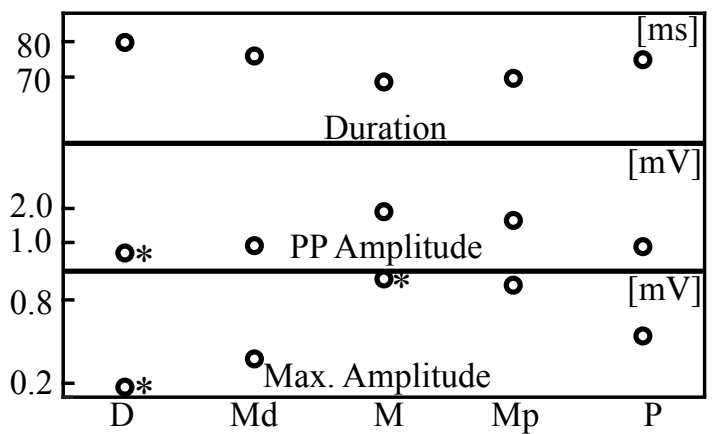

(a)

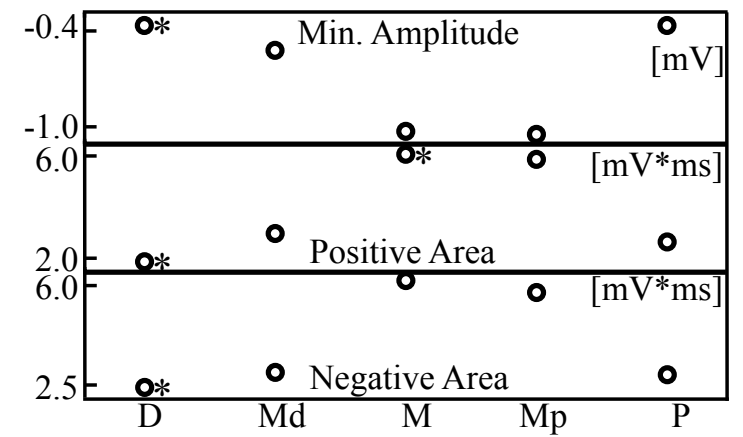

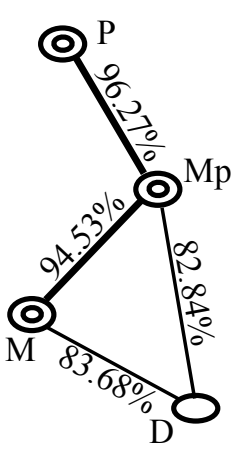

(b)

Figure 1. (a) Median of features for each channel. Asterisk shows statistically significant values. (b) Strongest (thick line) and weakest (thin line) correlations between channels. Channels with the highest correlations are shown with double circles. D: distal; Md: mid-distal; M: medial; Mp: mid-proximal; P: proximal.

Table 1. Statistical differences as $p$-values of KW test for multichannel analysis (first column) and MWUt for paired channels. D: distal; M: medial; Md: Mid-distal; Mp: Midproximal. Dur: duration; Amp: amplitude.

\begin{tabular}{lccccc}
\hline & KW & \multicolumn{4}{c}{ MWUt } \\
& & D-M & D-Mp & Md-M & Md-Mp \\
\hline \hline Dur & 0.0486 & 0.0071 & 0.0186 & 0.2064 & 0.3846 \\
Amp (+) & 0.0002 & 0.0001 & 0.0002 & 0.0213 & 0.0164 \\
Amp (-) & 0.0027 & 0.0014 & 0.0017 & 0.0275 & 0.0325 \\
Amp (PP) & 0.0054 & 0.0019 & 0.0033 & 0.0382 & 0.0413 \\
RMS & 0.0020 & 0.0007 & 0.0017 & 0.0195 & 0.0397 \\
Area (+) & 0.0027 & 0.0011 & 0.0022 & 0.0187 & 0.0447 \\
Area (-) & 0.0033 & 0.0016 & 0.0021 & 0.0223 & 0.0287 \\
Deflections & 0.7857 & 0.5189 & 0.4971 & 0.6562 & 0.7426 \\
Inflections & 0.5843 & 0.3109 & 1.0000 & 0.8613 & 0.2642 \\
\hline
\end{tabular}

channel ( $p=0.028)$ and shorter LAW duration in medial channel ( $p=0.035)$. Mid-proximal channel also showed a trend for the second highest amplitude and area values ( $p=0.022 \sim 0.063$ ), but not for duration. Difference between medial and distal channels was $14 \mathrm{~ms}$ in duration and $1.45 \mathrm{mV}$ in PP amplitude. Difference between medial and medial-proximal channels was $3.5 \mathrm{~ms}$ and $0.23 \mathrm{mV}$ in duration and amplitude, respectively. None of the channels showed any statistical difference in NODI features.

Figure 1.a shows how the medians of the most important features vary among channels, while Figure 1.b illustrates the strongest and weakest correlations between the dominant morphologies of LAWs recorded by catheter channels. It can be observed that the LAWs of mid-proximal channel show the highest correlation with LAWs of the adjacent channels. On the contrary, LAW morphology recorded by distal channel showed the lowest correlation with medial and mid-proximal channels.

\section{Discussion}

To our knowledge, this is the first study investigating the variation of fundamental LAW features across the channels of the CS recording catheter extracted from the CA process during SR. Results showed that the features of LAWs vary upon the employed channel, being distal and medial the channels that vary the most. More specifically, distal channel contained the lowest amplitude signals with longer LAW durations. On the other side, medial followed by mid-proximal, were the channels presenting the highest amplitude signals with LAWs of shorter duration. LAW morphology was also more common between medial to proximal channels and more diverse in distal than the rest of the channels.

The possibility of defective recordings provided by distal channel has been implied in previous studies, where distal or distal and proximal channels have been proved ineffective for the extraction of statistically significant results discriminating between groups of analysis [9, 20,21]. The results demonstrated in the present study are in agreement with these observations and provide a more detailed perspective on how choosing the wrong channel can affect the results of any work.

Although employing all available channels is the best strategy, sometimes it is not feasible due to external factors. In this case, channels which have been shown to provide the least altered information should be preferred. This study has shown medial and mid-proximal channels to preserve LAW features as intact as it could be, while distal followed by mid-distal and proximal channels to slightly alter duration and amplitude-based features.

\section{Conclusions}

A reliable AF substrate mapping requires the analysis of stable features in which the employment of medial and 
mid-proximal channels, which show the most preserved LAW information, is strongly suggested. On the other hand, distal is the least reliable channel with the most altered features and its employment should be avoided.

\section{Acknowledgments}

Research supported by grants DPI2017-83952-C3 from MINECO/AEI/FEDER UE，SBPLY/17/180501/000411 from JCCLM and AICO/2019/036 from GVA.

\section{References}

[1] Kirchhof P, Benussi S, Kotecha D, Ahlsson A, Atar D, Casadei B, Castella M, Diener HCea. 2016 ESC Guidelines for the management of atrial fibrillation developed in collaboration with EACTS. European Heart Journal 08 2016; 37(38):2893-2962. ISSN 0195-668X.

[2] Haïssaguerre M, Jaïs P, Shah DC, Takahashi A, Hocini M, Quiniou G, Garrigue S, Mouroux AL, Métayer PL, Clémenty J. Spontaneous initiation of atrial fibrillation by ectopic beats originating in the pulmonary veins. New England Journal of Medicine sep 1998;339(10):659-666.

[3] Ghzally Y, Gerasimon G. Catheter Ablation. StatPearls Publishing, 2020 Jan.

[4] Antz M, Otomo K, Arruda M, Scherlag BJ, Pitha J, Tondo C, Lazzara R, Jackman WM. Electrical conduction between the right atrium and the left atrium via the musculature of the coronary sinus. Circulation 1998;98(17):1790-1795.

[5] Chauvin M, Shah DC, Haïssaguerre M, Marcellin L, Brechenmacher $\mathrm{C}$. The anatomic basis of connections between the coronary sinus musculature and the left atrium in humans. Circulation 2000;101(6):647-652.

[6] Tada H, Yamada M, Naito S, Nogami A, Oshima S, Taniguchi K. Radiofrequency catheter ablation within the coronary sinus eliminates a macro-reentrant atrial tachycardia: Importance of mapping in the coronary sinus. Journal of Interventional Cardiac Electrophysiology Jan 2006; 15(1):35-41.

[7] Yoshida K, Ulfarsson M, Tada H, Chugh A, Good E, Kuhne M, Crawford T, Sarrazin JF, Chalfoun Nea. Complex electrograms within the coronary sinus: Time- and frequencydomain characteristics, effects of antral pulmonary vein isolation, and relationship to clinical outcome in patients with paroxysmal and persistent atrial fibrillation. Journal of Cardiovascular Electrophysiology ;19(10):1017-023.

[8] P. S, Marchlinski F. Techniques for the provocation, localization, and ablation of nonulmonary vein triggers for atrial fibrillation. Heart Rhythm 2017;14(7):1087 - 1096.

[9] Boles U, Gul EE, Enriquez A, Starr N, Haseeb S, Abdollah H, Simpson C, Baranchuk A, Redfearn Dea. Coronary sinus electrograms may predict new-onset atrial fibrillation after typical atrial flutter radiofrequency ablation (cse-af). Journal of atrial fibrillation Jun 2018;11(1):1809-1809.
[10] Tritto M, Zardini M, De Ponti R, Salerno-Uriarte JA. Iterative atrial tachycardia originating from the coronary sinus musculature. Journal of Cardiovascular Electrophysiology 2001;12(10):1187-1189.

[11] Rotter M, Sanders P, Takahashi Y, Hsu LF, Sacher F, Hocini M, Jaïs P, Haïssaguerre M. Coronary sinus tachycardia driving atrial fibrillation. Circulation 2004;110(6):e59-e60.

[12] Xia Y, Hertervig E, Kongstad O, Ljungström E, Pyotr P, Holm M, Olsson B, Yuan S. Deterioration of interatrial conduction in patients with paroxysmal atrial fibrillation: Electroanatomic mapping of the right atrium and coronary sinus. Heart Rhythm Nov 2004;1(5):548-553.

[13] Morita H, Zipes DP, Morita ST, Wu J. The role of coronary sinus musculature in the induction of atrial fibrillation. Heart Rhythm Apr 2012;9(4):581-589.

[14] Langenberg CJ, Pietersen HG, Geskes G, Wagenmakers AJ, Soeters PB, Durieux M. Coronary sinus catheter placement: Assessment of placement criteria and cardiac complications. CHEST October 2003;124(4):1259-1265.

[15] Mahmud E, Kerumati S, Raisinghani A. Dilation of the coronary sinus on echocardiogram: prevalence and significance. J Am Soc Echocardiography January 2001; 1(14):44-49.

[16] Martinez-Iniesta M, Ródenas J, Alcaraz R, Rieta JJ. Waveform integrity in atrial fibrillation: The forgotten issue of cardiac electrophysiology. Annals of biomedical engineering 2017;45(8):1890-1907.

[17] Botteron GW, Smith JM. A technique for measurement of the extent of spatial organization of atrial activation during atrial fibrillation in the intact human heart. IEEE Transactions on Biomedical Engineering 1995;42(6):579-586.

[18] Osorio D, Alcaraz R, Rieta JJ. A fractionation-based local activation wave detector for atrial electrograms of atrial fibrillation. In 2017 Computing in Cardiology (CinC). IEEE, 2017; $1-4$.

[19] Alcaraz R, Hornero F, Martínez A, Rieta JJ. Short-time regularity assessment of fibrillatory waves from the surface ecg in atrial fibrillation. Physiological measurement 2012; 33(6):969.

[20] Teh AW, Kalman JM, Kistler PM, Lee G, Sutherland F, Morton JB, et al. Prevalence of fractionated electrograms in the coronary sinus: comparison between patients with persistent or paroxysmal atrial fibrillation and a control population. Heart Rhythm 2010;7(9):1200-1204.

[21] Di Marco LY, Raine D, Bourke JP, Langley P. Characteristics of atrial fibrillation cycle length predict restoration of sinus rhythm by catheter ablation. Heart Rhythm 2013; 10(9):1303-1310.

Address for correspondence:

José J. Rieta

BioMIT.org, Electronic Engineering Department, Building 7F-5 Universitat Politecnica de Valencia, 46022 Valencia, Spain.

e-mail: jjrieta@upv.es 